\title{
A simple synthesis and characterization of CuS nanocrystals
}

\author{
UJJAL K GAUTAM* and BRATINDRANATH MUKHERJEE ${ }^{\dagger}$ \\ Solid State and Structural Chemistry Unit, ${ }^{\dagger}$ Materials Research Centre, Indian Institute of Science, \\ Bangalore 560 012, India
}

MS received 12 July 2005; revised 8 November 2005

\begin{abstract}
Water-soluble CuS nanocrystals and nanorods were prepared by reacting copper acetate with thioacetamide in the presence of different surfactants and capping agents. The size of the nanocrystals varied from 3-20 nm depending on the reaction parameters such as concentration, temperature, solvent and the capping agents. The formation of nanocrystals was studied by using UV-visible absorption spectroscopy.
\end{abstract}

Keywords. Copper sulfide; nanocrystals; solution synthesis.

\section{Introduction}

Synthesis and characterization of nanocrystals of semiconducting metal sulfides have been an intense field of research due to their interesting properties and potential applications (Murray et al 2000; Hu et al 2001; Trinidade et al 2001; Rao et al 2004; Rotello 2004). For example, when the size of the nanocrystals is smaller than the Bohr excitation radius of the material, they exhibit properties which are size dependent and distinct from the bulk (Alivisatos 1996). Such materials are promising candidates in electronics, data storage, energy storage, catalysis and sensors (Heath et al 1998; Huynh et al 2002; Kung and Kung 2004). It is, therefore, important to develop synthetic strategies which are simple, cost-effective, environment friendly, easily scalable and at the same time with parameters to control size and shape of the materials. CuS has potential applications in solar cells, IR detectors and lubrication (Mane and Lokhande 2000). It exhibits fast-ion conduction at high temperature (Paul et al 1993). However, copper sulfide has a fairly complex crystal chemistry owing to its ability to form sub-stoichiometric compounds, $\mathrm{Cu}_{x} \mathrm{~S}(2 \geq x \geq 1)$ (Evans 1971, 1979; Grijavala et al 1996). The CuS phase exists in two forms, the amorphous brown $\mathrm{CuS}$ and the green crystalline covellite (Brelle et al 2000). Traditionally $\mathrm{CuS}$ is prepared by solid state reactions (Parkin 1996). There are a few reports of solution synthesis as well (Silvester et al 1991; Haram et al 1996; Henshaw et al 1997; Jiang et al 2000; Dong et al 2002; Wang et al 2002; Lu et al 2003; Zhang et al 2004). Jiang and coworkers (2000) prepared nanocrystalline $\mathrm{Cu}_{9} \mathrm{~S}_{8}, \mathrm{Cu}_{7} \mathrm{~S}_{4}$ and $\mathrm{CuS}$ solvothermally by controlling the release of $\mathrm{S}^{2-}$ from thiourea. Dong et al (2002) used water-in-carbon dioxide micro-emulsion to prepare $\mathrm{CuS}$ nanocrystals.

\footnotetext{
*Author for correspondence (gautam@sscu.iisc.ernet.in)
}

Others involve micro-emulsion synthesis using non-ionic Tritin X100 surfactant, sonochemical technique and elemental reactions in liquid ammonia (Haram et al 1996; Henshaw et al 1997; Wang et al 2002). Sylvester and coworkers (1991) investigated the kinetics of formation of $\mathrm{Cu}_{x} \mathrm{~S}$ from $\mathrm{Cu}^{2+}$ salt and $\mathrm{H}_{2} \mathrm{~S}$ gas in detail. Nanorods of $\mathrm{CuS}$ have also been prepared by using liquid-crystal templates (Lu et al 2003). Notably, Larsen et al (2003) reported a solventless synthesis of $\mathrm{Cu}_{2} \mathrm{~S}$ nanorods, by heating copper thiolate to high temperatures. Recently, we have obtained single crystalline ultra-thin films of $\mathrm{CuS}$ prepared by a novel technique involving reactions at the liquidliquid interface (Gautam et al 2004). However, in view of its usefulness, there is a need to evolve environmentally friendly routes to prepare $\mathrm{CuS}$ nanocrystals, which yields nanocrystals of desired sizes in a controllable manner.

In this article, we describe a facile aqueous synthesis of $\mathrm{CuS}$ nanocrystals of various sizes and shapes. The effects of various reaction parameters on the size of the nanoparticles have been examined. Use of surfactants renders stability to the nanocrystals for months against ambient oxidation and corrosion. Importantly, the present strategy avoids toxic chemicals either in the form of reactants or as side products.

\section{Experimental}

Copper acetate monohydrate $\left(\mathrm{Cu}(a c)_{2}\right)$ and thioacetamide $\left(\mathrm{CH}_{3} \mathrm{CSNH}_{2}\right)$ were used as $\mathrm{Cu}$ and sulfur sources, respectively for all the reactions. In a typical preparation, $0.0050 \mathrm{~g}$ of $\mathrm{Cu}(a c)_{2}(25 \mu \mathrm{mol})$ was dissolved in $10 \mathrm{ml}$ deionized water. In another container, $0.0020 \mathrm{~g}$ of thioacetamide (25 $\mu \mathrm{mol})$ and $0.200 \mathrm{~g}$ of sodium-bis(2-ethylhexyl) sulfosuccinate (Na-AOT) were dissolved in $10 \mathrm{ml}$ of deionized water. The $\mathrm{Cu}(a c)_{2}$ solution was added to the thioacetamide solution dropwise at $30^{\circ} \mathrm{C}$ with continuous stirring 
over a period of $20 \mathrm{~min}$. The solution turned golden brown as soon as the first drop of $\mathrm{Cu}(a c)_{2}$ solution was added and the colour deepened with more addition. The brown solution turned green over a period of $12 \mathrm{~h}$. Cetyl trimethylammonium bromide (CTAB) capped nanocrystals were prepared in a similar fashion, using $0.100 \mathrm{~g}$ of CTAB instead of Na-AOT. Similarly, polyvinyl pyrrolidon (PVP) capped nanocrystals were prepared in methanol medium instead of water, keeping all other conditions identical. The green solutions containing $\mathrm{CuS}$ nanocrystals were examined by transmission electron microscopy (TEM), electronic absorption spectroscopy and X-ray diffraction technique. A drop of the solution was dried on a holey carbon grid and investigated using a JEOL (JEM3010) transmission electron microscope operating with an accelerating voltage of $300 \mathrm{kV}$. The solution was dried on a glass slide in order to record the X-ray diffraction pattern on a Siemens 5005 Diffractometer with reflection BraggBrentano geometry using $\mathrm{CuK} \alpha$ radiation $(\lambda=1.5418 \AA$ ). $\mathrm{UV}$-vis absorption spectra of the nanocrystals in water were recorded using a Perkin-Elmer UV-visible spectrometer.

\section{Results and discussion}

In figure 1a, we show a typical TEM image of $11 \mathrm{~nm} \mathrm{CuS}$ nanocrystals obtained by reacting 1.25 mmolar solution, $\mathrm{Cu}(a c)_{2}$, with 1.25 mmolar solution of thioacetamide. The histogram in the inset shows the particle size distribution. The nanocrystals are not perfectly spherical and the particle size indicates average of the horizontal and vertical diameters. The nanocrystals are stable in solution for long periods, due to surface passivation by the surfactant and do not precipitate even after months. However, the TEM images reveal that the particles tend to agglomerate to some extent. This is probably due to the drying artifact during the preparation of the grid for TEM imaging. The top inset in figure 1a shows the selected area diffraction pattern (SAED) of the nanocrystals. The pattern can be indexed on hexagonal CuS. In figure $2 \mathrm{a}$, we show the X-ray diffraction pattern of the $11 \mathrm{~nm}$ nanocrystals obtained at $30^{\circ} \mathrm{C}$. All the peaks in the pattern correspond to phase pure $\mathrm{CuS}$ in the space group $P 6_{3} / m m c$ (JCPDF no. 03-0724). Figure $2 b$ shows electronic absorption spectrum of the
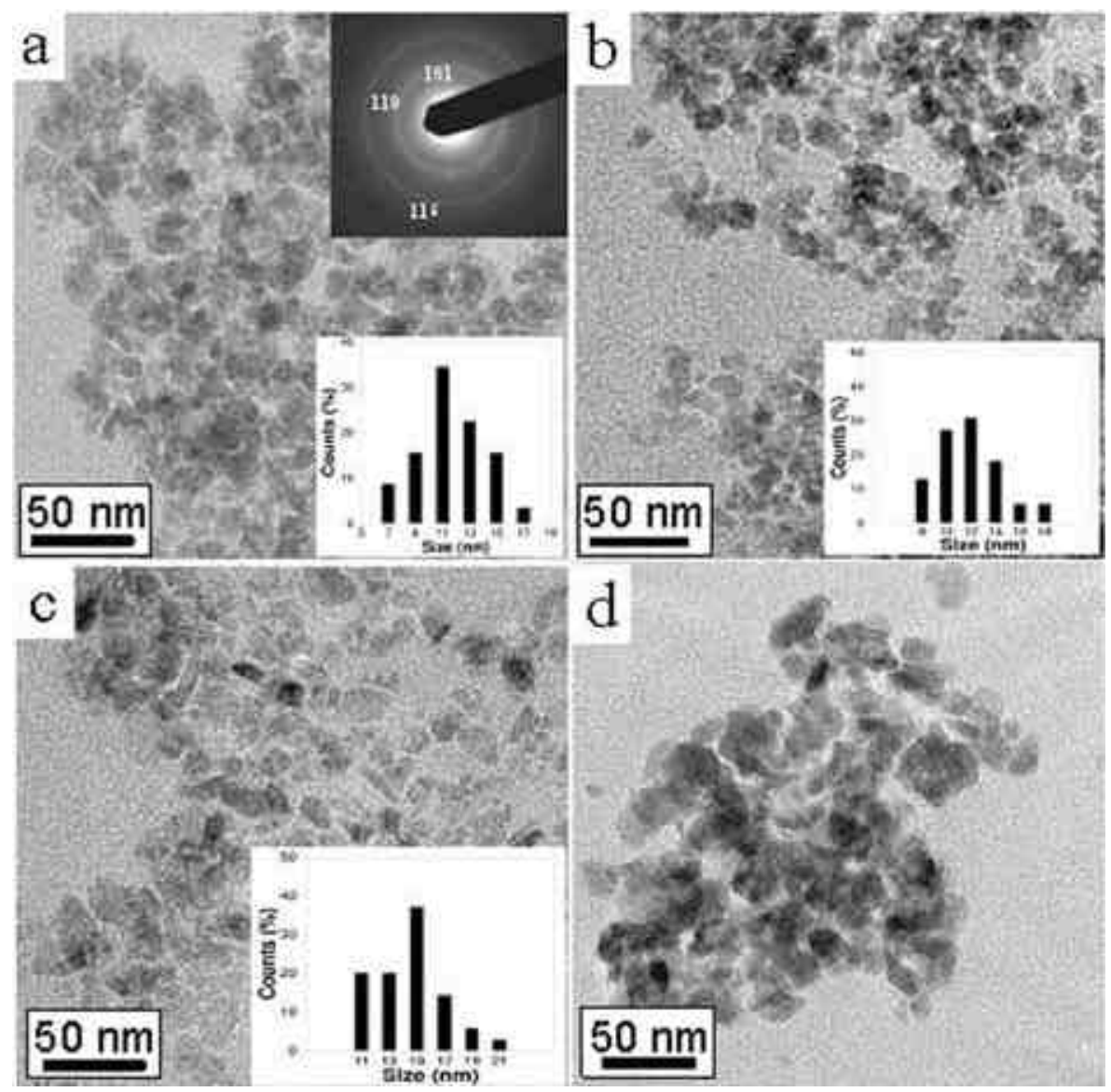

Figure 1. TEM images of the CuS nanocrystals obtained by using a. $12.5 \mu \mathrm{mol}$ of $\mathrm{Cu}(a c)_{2}$ $(11 \mathrm{~nm})$, b. $25 \mu \mathrm{mol}$ of $\mathrm{Cu}(a c)_{2}(12 \mathrm{~nm})$, c. $37.5 \mu \mathrm{mol}$ of $\mathrm{Cu}(a c)_{2}(15 \mathrm{~nm})$ and d. reaction temperature of $15^{\circ} \mathrm{C}(20 \mathrm{~nm})$. 

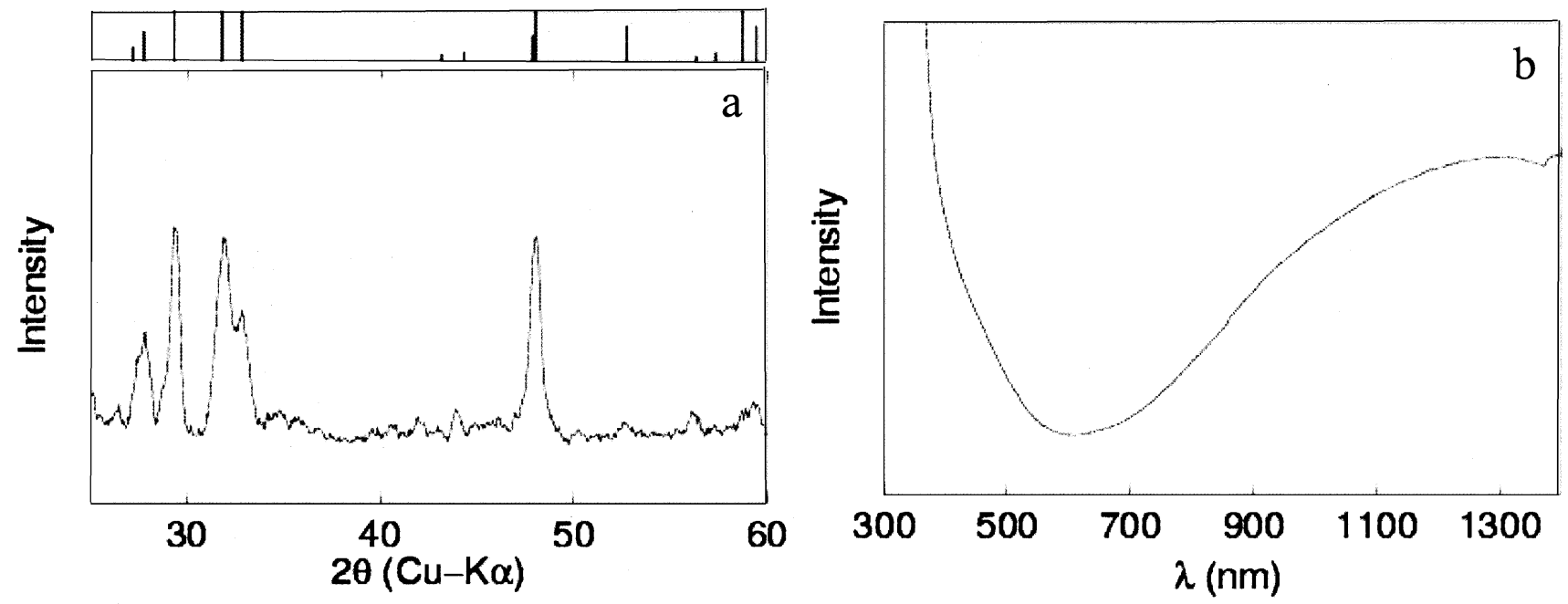

Figure 2. a. The XRD pattern of $11 \mathrm{~nm} \mathrm{CuS} \mathrm{nanocrystals.} \mathrm{The} \mathrm{upper} \mathrm{panel} \mathrm{shows} \mathrm{the} \mathrm{expected} \mathrm{peak} \mathrm{positions} \mathrm{and} \mathrm{b.} \mathrm{the} \mathrm{corre-}$ sponding UV-vis absorption spectrum of the nanocrystals.
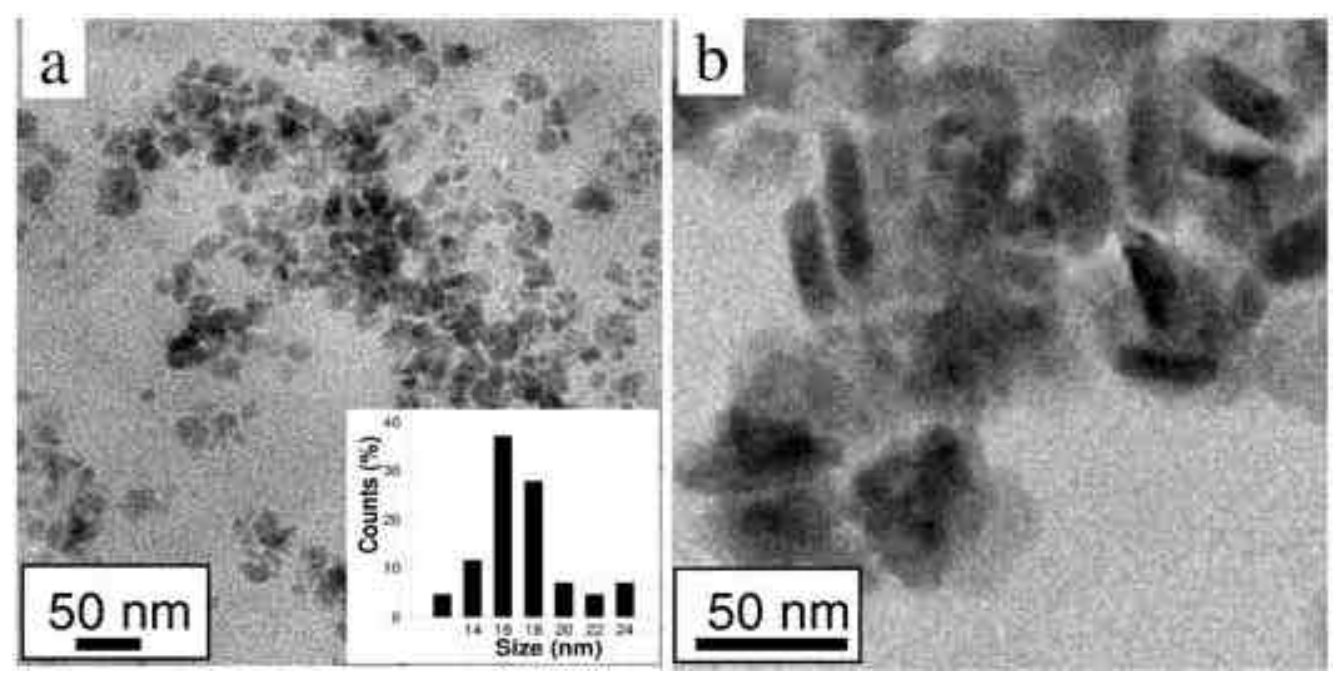

Figure 3. a. $16 \mathrm{~nm} \mathrm{CuS} \mathrm{nanocrystals} \mathrm{obtained} \mathrm{reducing} \mathrm{the} \mathrm{amount} \mathrm{of} \mathrm{NaAOT} \mathrm{by} \mathrm{half} \mathrm{and} \mathbf{b} . \mathrm{CuS}$ nanorods obtained in presence of excess NaAOT.

nanocrystals. Eventhough $\mathrm{Cu}_{x} \mathrm{~S}$ has many stable phases with varied stoichiometry, each phase has its distinct optical absorption spectrum. The broad absorption in the near IR region corresponds to the pure covellite phase (Silvester et al 1991; Yumashev et al 1996). We do not observe any shoulder at $\sim 450 \mathrm{~nm}$ corresponding to the $\mathrm{Cu}_{2} \mathrm{~S}$ phase (Haram et al 1996).

The particle size of $\mathrm{CuS}$ increases with the reactant concentrations. For example, $12 \mathrm{~nm}$ and $15 \mathrm{~nm}$ nanocrystals were observed by increasing the concentrations of all the reactants two-fold and four-fold, respectively (figures $1 \mathrm{~b}$ and c). The size of the nanocrystals is also dependent on the reaction temperature. We obtained larger nanocrystals of $20 \mathrm{~nm}$ (compared with the $12 \mathrm{~nm}$ particles) with a broad size distribution by carrying out the preparation at $15^{\circ} \mathrm{C}$ (see figure $1 \mathrm{~d}$ ). However, the product obtained at $50^{\circ} \mathrm{C}$ had large agglomerates of $8-12 \mathrm{~nm}$ nanocrystals. Fast production of a large number of nuclei at $50^{\circ} \mathrm{C}$ appears to result in smaller nanocrystals which get attached to one another due to high temperature.

The concentration of Na-AOT influences size and shape of the $\mathrm{CuS}$ nanocrystals. We obtained $16 \mathrm{~nm}$ nanocrystals of $\mathrm{CuS}$ (figure $3 \mathrm{a}$ ) by using $0 \cdot 100 \mathrm{~g}$ of the surfactant as compared to the $12 \mathrm{~nm}$ nanocrystals obtained by using $0 \cdot 200 \mathrm{~g}$ as shown in figure $1 \mathrm{a}$. By increasing the amount of Na-AOT to $0 \cdot 300 \mathrm{~g}$, rod shaped $\mathrm{CuS}$ nanocrystals were obtained as shown in figure $3 \mathrm{~b}$.

$\mathrm{CuS}$ nanocrystals of much smaller diameter were obtained by using methanol solvent along with PVP cap and CTAB cap in the aqueous medium. Figure 4 a displays the 

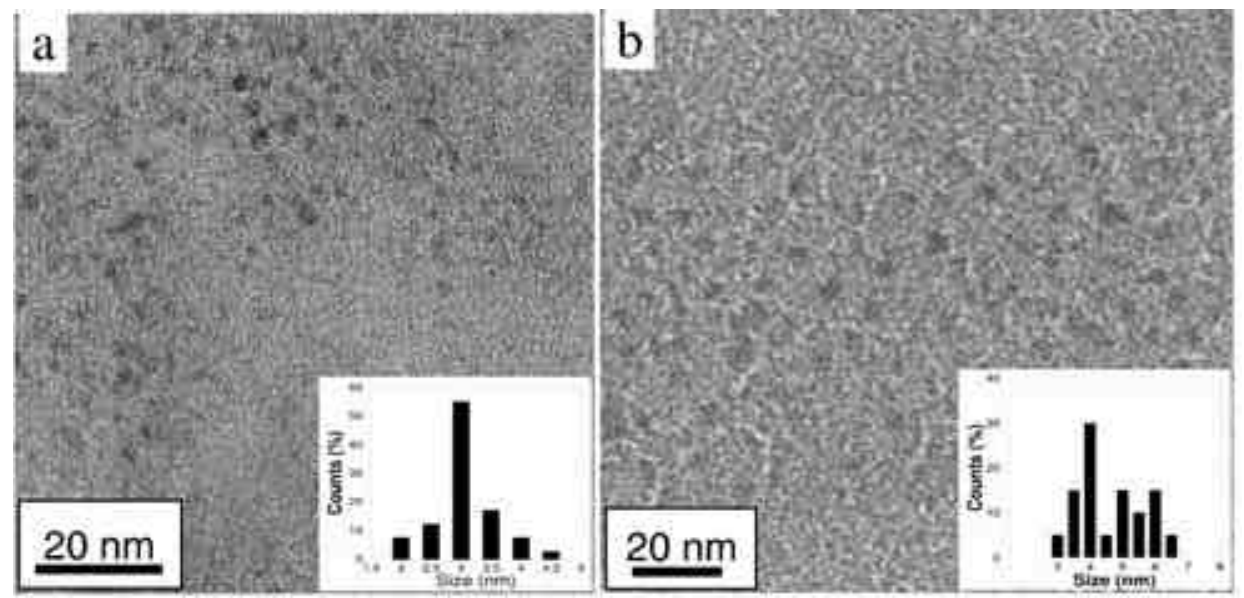

Figure 4. a. $3 \mathrm{~nm} \mathrm{CuS}$ nanocrystals obtained in methanol solvent and b. $4 \mathrm{~nm} \mathrm{CuS}$ nanocrystals obtained in an aqueous synthesis when CTAB was used as capping agent in aqueous medium.

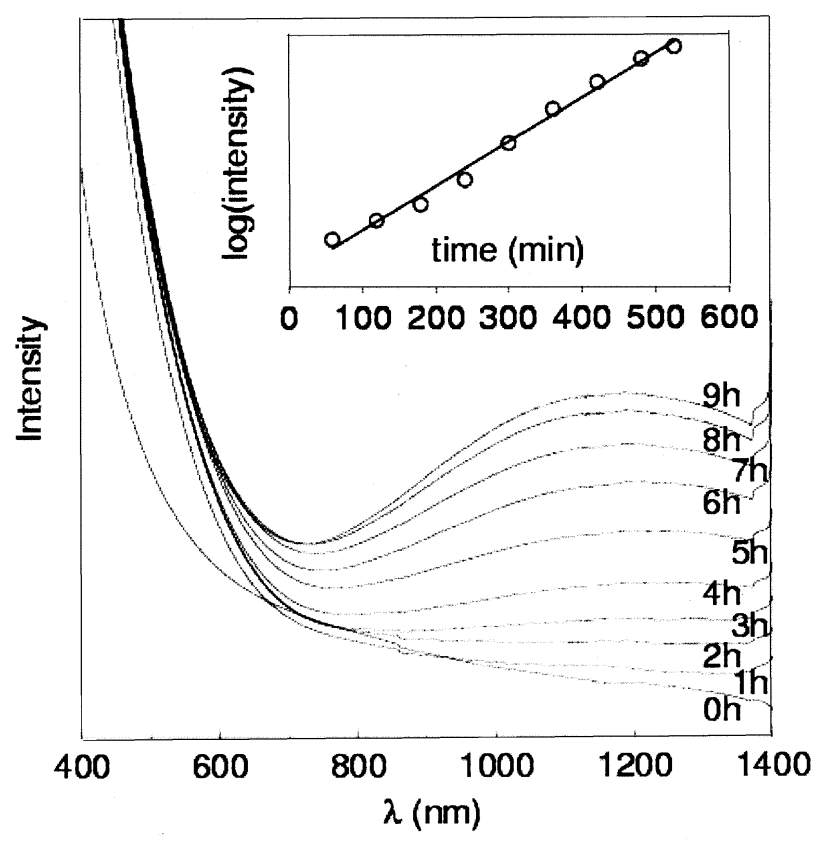

Figure 5. The UV-vis absorption spectra of the CuS nanocrystals recorded at $1 \mathrm{~h}$ interval as they form. The inset shows the $\log$ of intensity of near-IR absorption band vs time of the reaction.

$3 \mathrm{~nm} \mathrm{CuS} \mathrm{nanocrystals} \mathrm{obtained} \mathrm{thus} \mathrm{in} \mathrm{methanol} \mathrm{solvent,}$ keeping concentrations of all the chemicals same as the sample shown in figure $1 \mathrm{~b}$. Part of the nanocrystals precipitated out, the rest remaining in the solution imparting green colour. Figure $4 \mathrm{~b}$ shows $4 \mathrm{~nm} \mathrm{CuS}$ nanocrystals obtained by using $0 \cdot 10 \mathrm{~g}$ of CTAB.

The kinetics of formation of $\mathrm{CuS}$ nanocrystals have been reported by Silvester et al (1991), who employed gaseous $\mathrm{H}_{2} \mathrm{~S}$ as one of the reactants. We now examine the growth of nanocrystals, keeping all the reactants in solution phase. The near IR absorption band in the absorption spectra of $\mathrm{CuS}$ nanocrystals provides a good means to study the growth process. This is because the band does not shift with time and its intensity is directly proportional to the amount of $\mathrm{CuS}$ in the solution. In figure 5 we show the $\mathrm{UV}$-vis spectra of the CuS nanocrystals (for the sample in figure $1 \mathrm{~b}$ ) recorded at intervals of $1 \mathrm{~h}$. As can be seen, the band is absent when the two solutions are just mixed and gradually grows with time. The inset in the figure displays the plot of log of intensity of the band against time showing a near linear growth of the band.

\section{Conclusions}

We have prepared $\mathrm{CuS}$ nanocrystals of various sizes and shapes by a simple and environmentally benign technique involving the reaction of copper acetate with thioacetamide. The reaction carried out in polar organic solvent in presence of CTAB yielded 3-4 nanocrystals while that in aqueous medium yielded $11-20 \mathrm{~nm}$ nanocrystals. The technique is cost-effective and scalable.

\section{Acknowledgement}

We thank Prof. C N R Rao for suggesting the problem and helpful discussions.

\section{References}

Alivisatos A P 1996 Science 271933

Brelle M C, Martinez C L T, McNulty J C, Mehra R K and Zhang J Z 2000 Pure Appl. Chem. 72101

Dong X, Potter D and Erkey C 2002 Ind. Eng. Chem. Res. 41 4489

Evans H T Jr 1971 Nature (London) Phys. Sci. 23269

Evans T 1979 Science 203356 
Gautam U K, Ghosh M and Rao C N R 2004 Langmuir 2010775 Grijavala H, Inoue M, Buggavaraou S and Calvert P 1996 J. Mater. Chem. 61157

Haram S K, Mahadeshwar A R and Dixit S G 1996 J. Phys. Chem. 1005868

Heath R, Kuekes P J, Snider G S and Williams R S 1998 Science 2801716

Henshaw G, Parkin I P and Shaw G A 1997 J. Chem. Soc. Dalton Trans. 231

Hu J, Li L, Yang W, Manna L, Wang L and Alivisatos A P 2001 Science 2922060

Huynh W U, Dittmer J J and Alivisatos A P 2002 Science 295 2425

Jiang X, Xie Y, Lu J, He W, Zhu L and Qian Y 2000 J. Mater. Chem. 102193

Kung H H and Kung M C 2004 Catalysis Today 97219

Larsen T H, Sigman M, Ghezelbash A, Doty R C and Korgel B A 2003 J. Am. Chem. Soc. 1255638

Lu J, Zhao Y, Chen N and Xie Y 2003 Chem. Lett. 3230

Mane R S and Lokhande C D 2000 Mater. Chem. Phys. 651
Murray C B, Kagan C R and Bawendi M G 2000 Ann. Rev. Mater. Sci. 30545

Parkin I P 1996 Chem. Soc. Rev. 25199

Paul P P, Rauchfuss T B and Wilson S R 1993 J. Am. Chem. Soc. 1153316

Rao C N R, Mueller A and Cheetham A K (eds) 2004 The chemistry of nanomaterials (Weinheim: Wiley-VCH)

Rotello V (ed.) 2004 Nanoparticles: Building blocks for nanotechnology (New York: Kluwer Academic Press)

Silvester E J, Grieser F, Sexton B A and Healy T W 1991 Langmuir 72917

Trinidade T, O'Brien P and Pickett N L 2001 Chem. Mater. 13 3843

Wang H, Zhang J R, Zhao X N, Xu S and Zhu J J 2002 Mater. Letts $\mathbf{5 5} 253$

Yumashev K V, Prokoshin P V, Malyarevich A M, Mikhailov V P, Artemyev M V and Gurin V S 1996 Appl. Phys. B64 73

Zhang Y C, Hu X Y and Quao T 2004 Solid State Commun. 132 779 